\title{
The benefits evaluation of abdominal deep inspiration breath hold based on knowledge-based radiotherapy treatment planning for left-sided breast cancer
}

\section{Jiaqi Xu}

Zhejiang University School of Medicine First Affiliated Hospital

Jiazhou Wang

Fudan University Shanghai Cancer Center

\section{Feng Zhao}

Zhejiang University School of Medicine First Affiliated Hospital

\section{Weigang $\mathrm{Hu}$}

Fudan University Shanghai Cancer Center

\section{Luyi Bu}

Zhejiang University School of Medicine First Affiliated Hospital

\section{Guorong Yao}

Zhejiang University School of Medicine First Affiliated Hospital

Zhongjie Lu ( $\sim$ luzhongjie@zju.edu.cn )

Zhejiang University School of Medicine First Affiliated Hospital

\section{Senxiang Yan}

Zhejiang University School of Medicine First Affiliated Hospital

\section{Research}

Keywords: Deep inspiration breath hold, dose distribution prediction, knowledge-based planning, machine learning, breast cancer

Posted Date: March 5th, 2020

DOI: https://doi.org/10.21203/rs.3.rs-16183/v1

License: (9) (1) This work is licensed under a Creative Commons Attribution 4.0 International License. Read Full License 
Version of Record: A version of this preprint was published at Journal of Applied Clinical Medical Physics on September 12th, 2020. See the published version at https://doi.org/10.1002/acm2.13013. 
1 The benefits evaluation of abdominal deep inspiration breath hold

2 based on knowledge-based radiotherapy treatment planning for

3 left-sided breast cancer

4

5 Jiaqi $\mathrm{Xu}^{\mathrm{a}}$, Jiazhou $\mathrm{Wang}^{\mathrm{b}}$, Feng Zhao ${ }^{\mathrm{a}}$, Weigang $\mathrm{Hu}^{\mathrm{b}}$, Luyi $\mathrm{Bu}^{\mathrm{a}}$, Guorong $\mathrm{Yao}^{\mathrm{a}}$,

6 Zhongjie $\mathrm{Lu}^{\mathrm{a}^{*}}$, Senxiang Yan ${ }^{\mathrm{a}^{*}}$

$7{ }^{\text {a }}$ Department of Radiation Oncology, the First Affiliated Hospital, College of

8 Medicine, Zhejiang University, Hangzhou, Zhejiang, 310003, China

9 b Department of Radiation Oncology, Fudan University Shanghai Cancer Center, 10 Shanghai, 200032, China

11 * Corresponding author: Zhongjie Lu, Senxiang Yan 


\section{Abstract}

Purpose: Study the impact of abdominal deep inspiration breath hold (DIBH) technique on knowledge-based radiotherapy treatment planning for left-sided breast cancer to guide the application of DIBH radiotherapy technology.

Methods and Materials: Two kernel density estimation (KDE) models were developed based on 40 left-sided breast cancer patients with two CT acquisitions of free breathing (FB-CT) and DIBH (DIBH-CT). Each KDE model was used to predict DVHs based on DIBH-CT and FB-CT for another 10 new patients similar to our training datasets. The predicted DVHs were taken as a substitute to dose constraints and objective functions in the Eclipse treatment planning system, with the same requirements for the planning target volume (PTV). The mean doses to the heart, the left anterior descending coronary artery (LADCA) and the ipsilateral lung were evaluated and compared using the T-test among clinical plans, KDE predictions, and KDE plans.

Results: Our study demonstrated that the KDE model can generate deliverable simulations equivalent to clinically applicable plans. The T-test was applied to test the consistency hypothesis on another 10 left-sided breast cancer patients. In cases of the same breathing status, there was no statistically significant difference between the predicted and the clinical plans for all clinically relevant dose volume histogram (DVH) indices ( $>>0.05)$, and all predicted DVHs can be transferred into deliverable plans. For DIBH-CT images, significant differences were observed in $\mathrm{D}_{\text {mean }}$ between FB model predictions and the clinical plans $(\mathrm{p}<0.05)$. DIBH model prediction cannot 

56

be optimized to a deliverable plan based on FB-CT, with a counsel of perfection.

Conclusion: This study demonstrated that the KDE prediction results were well fitted for the same breathing condition but degrade with different breathing conditions. The benefits of DIBH can be evaluated quickly and effectively by the specific knowledge-based treatment planning for left-sided breast cancer radiotherapy. This study will help to further realize the goal of automatic treatment planning.

Keywords: Deep inspiration breath hold, dose distribution prediction, knowledge-based planning, machine learning, breast cancer

(1)

\section{5}

(1)

\section{Background}

Postoperative adjuvant radiotherapy (RT) plays an indispensable role in breast-conserving treatment to minimize the risks of local-regional recurrence and metastasis. Whole breast irradiation (WBI) after breast-conserving surgery as a comprehensive treatment model, which has been confirmed to possess the similar local control and overall survival rates to modified radical surgery in breast cancer patients ${ }^{[1]}$. However, the dose of the surrounding critical organs-at-risks (OARs), especially the heart, left lung and the left anterior descending coronary artery $(\text { LADCA })^{[2-4]}$, are crucial to the RT quality assessment for left-sided breast cancer. Therefore, by using diversity of methods, such as DIBH, intensity-modulated 
radiation therapy (IMRT) techniques, treatment in the prone position and proton therapy, to shield the heart and minimize the lung and LADCA doses while ensuring enough dose in the target volume during left breast postoperative radiotherapy have been presented. Combining DIBH and IMRT is the most commonly-used strategy in left-sided breast postoperative radiotherapy ${ }^{[5-7]}$. The DIBH maneuver we used is the abdominal DIBH (A-DIBH), which could widen the spatial Euclidean distances between the heart and the target volume. IMRT treatment technique has the capability of reducing the cardiac dose while delivering adequate target coverage because of its unique dose calculation and beam weight optimization.

The selection of the final radiotherapy regimen (especially the selection of respiratory mode) will greatly affect the normal tissue complications (NTCP) and tumor control rate ${ }^{[8,9]}$. In the past IMRT plans, physicians often determined the ideal OARs dose-volume limit through population-based recommendations (either from the tumor radiotherapy team or from the doctor's intuition) ${ }^{[10]}$. However, the huge geometric differences in the complexity of PTV and OAR among patients make it a challenge for doctors to quickly and accurately select the best ultimate treatment for a particular patient within all acceptable options.

Knowledge-based planning $(\mathrm{KBP})$ is a promising technology. There is a large amount of image information and dose planning information of cancer patients in the current radiotherapy system, which has become a priori knowledge. By feature extraction and quantitative analysis of these prior knowledge, a reliable empirical model (KBMs) can be obtained to realize the intelligence of the radiotherapy planning 
system. Current studies have proved that KBP has a higher consistency of plan quality and higher operational efficiency than manual plans with different quality ${ }^{[11-13]}$. For example, RapidPlan ${ }^{\mathrm{TM}}$ (Varian Medical Systems, Palo Alto, CA, USA) has been widely used as a commercial KBP product ${ }^{[11,14]}$.

In the KBP method, the prediction of DVH in new patients requires the use of the DVH of OAR in the previous clinical plan and the parameterized model generated by the relevant anatomical structure ${ }^{[13,17]}$, thus emphasizing the importance of the parameterized prior model. However, it remains to be seen whether the implementation effect of the parameterized prior model in KBP is consistent under different breathing conditions. To our best knowledge, the impacts of different breathing methods during CT simulation for left-sided breast cancer on knowledge-based treatment planning have not been reported before.

Therefore, this study established two knowledge-based empirical models for the treatment of the same group of breast cancer patients based on different respiratory conditions. We then used these two KBMs to cross-predict CT in both breathing patterns, creating four KBP plans for each patient. We attempted to investigate the compatibility of KBP with different respiratory conditions, such as whether the DIBH $\mathrm{KBM}$ is applicable to FB-CT prediction, or whether the FB KBM is applicable to DIBH-CT prediction. Quantifying the benefits of using the specified model can help us clearly understand the use of KBM to predict the OAR dose of postoperative radiotherapy for breast cancer and guide the application of A-DIBH radiotherapy technology. 


\section{Methods}

The workflow of this study is illustrated in the diagram on Figure 1. Firstly, two KBMs (FB model and DIBH model) were built from 40 existing clinical plans that have ideal tumor coverage and OAR doses with different breath settings. Another 10 new patients were selected to investigate the performance of the prediction model. Two treatment plans were established with uniform standards by the experienced physicist, and then confirmed by another senior physicist.

DVHs of these 10 new patients were estimated by two KBMs. The estimated DVHs were taken as a substitute to dose constraints and objective functions in Eclipse treatment planning system (TPS) for each new patient.

There are three types of comparisons we want to investigate in this study. Firstly, we want to confirm whether our model can precisely predict the DVHs with same breath settings, such as using FB model to predict patients with FB CT. These comparisons were marked as 'green' in Figure 1.

Secondly, we want to investigate whether model build with one breath condition can precisely predict DVH for patients with another breath. Such as using FB model to predict the DVH for the patient with DIBH CT images. These comparisons were marked as 'blue' in Figure 1.

Third, we want to investigate whether these DVH prediction models can be optimized to a deliverable plan. These comparisons were marked as 'red' in Figure1. 


\begin{tabular}{|c|c|c|c|}
\hline$\frac{\text { THE WORKFLOW OF THIS }}{\text { STUDY }}$ & \multicolumn{3}{|c|}{ Performance eVAluation } \\
\hline \multirow{3}{*}{\begin{tabular}{|c|} 
Two CT scans \\
(FB and DIBH)
\end{tabular}} & & FB model & DIBH model \\
\hline & \multirow{2}{*}{ FB-CT } & $\begin{array}{l}\text { Clinical plan VS. } \\
\text { KDE prediction }\end{array}$ & $\begin{array}{l}\text { Clinical plan VS. } \\
\text { KDE prediction }\end{array}$ \\
\hline & & $\begin{array}{l}\text { KDE plan VS. } \\
\text { KDE prediction }\end{array}$ & $\begin{array}{l}\text { KDE plan VS. } \\
\text { KDE prediction }\end{array}$ \\
\hline prediction & \multirow{2}{*}{ DIBH-CT } & $\begin{array}{l}\text { Clinical plan VS. } \\
\text { KDE prediction }\end{array}$ & $\begin{array}{l}\text { Clinical plan VS. } \\
\text { KDE prediction }\end{array}$ \\
\hline $\begin{array}{l}\text { Performance } \\
\text { evaluation }\end{array}$ & & $\begin{array}{l}\text { KDE plan VS. } \\
\text { KDE prediction }\end{array}$ & $\begin{array}{l}\text { KDE plan VS. } \\
\text { KDE prediction }\end{array}$ \\
\hline
\end{tabular}

Figure 1: The strategy of knowledge-based planning generated optimization objectives

\section{Patients and treatment planning}

The training dataset consisted of 40 consecutive previously administered by adjuvant RT following breast-conserving surgery for the left-side breast carcinoma. The mean age of those patients was 45.7 (range, 28-70), and the median age was 49 . Each patient underwent two CT simulation scans with a Siemens Sensation Open 24-slice scanner (Siemens, Forchheim, Germany): the FB condition and the A-DIBH condition. For consistency, all patients were practiced A-DIBH according to audio and visual coaching for at least a week before the simulation scan, until they can repeat the mode and hold their breath for 15-20s with the auxiliary of Varian Real-time Position Management (RPM) System.

To achieve optimal homogeneity of the data in the present analysis, we incorporated only the whole-breast irradiation series. Target volumes and OAR were entirely contoured via two CT series in the Eclipse treatment planning system (Varian Medical Systems, Palo Alto, CA, USA) according to the Danish Breast Cancer Cooperative Group (DBCG) atlas ${ }^{[18]}$. Two intensity modulated radiotherapy treatment plans were generated in the Eclipse for each CT, using the anisotropic analytical 
algorithm (AAA) for final dose calculation. All IMRT plans containing 6 fixed non-opposing fields with the same gantry angles and beam energies as the clinical methods.

The criterion of treatment plans was that $97 \%$ of the PTV should be covered by at least $95 \%$ of the isodose (and $<108 \%$ of the isodose), and the mean dose of PTV in the whole cases was prescribed to 50 Gy in 25 fractions.

\section{KDE Model training}

Inspired by Skarpman's KDE algorithm ${ }^{[17]}$, two KDE prediction models were developed based on 40 left-sided breast cancer patients with two different CT scans of FB and DIBH in the aforementioned high-quality IMRT cases. These cases were planned by the experienced dosimetric and approved for clinical treatment by attending physicians. Each KBM was applied to its training dataset then. The estimated DVH was compared with the clinical DVH to verify the reliability of the KDE model.

\section{DVH prediction and plan optimization}

Another 10 patients similar to our training datasets were enrolled for different model evaluation. Each patient has two images, FB-CT and DIBH-CT. The DVHs of each image was estimated by two KDE models, FB and DIBH model. So, each patient has four estimated DVHs, marked as KDE predictions.

To demonstrate whether these estimated DVHs can be directly used to generate deliverable plans, we created another four plans based on four cross-estimated DVHs.

We use the estimated DVHs generated in the previous step as dose constraints and 
objective functions at the specific points without any additional auxiliary human intervention (Table 1). The KDE plan was optimized on the Eclipse treatment planning system then. The gantry angles of these plans were exactly the same as the original clinical plans.

Table 1: OAR dose constraints points used for plan optimization for $200 \mathrm{~Gy} / \mathrm{fx}$ plan in 25 fractions.

\begin{tabular}{|c|c|c|c|c|c|c|}
\hline Organ & & $\overline{\text { Dos }}$ & const & ints $\mathrm{p}$ & & \\
\hline Heart & $\mathrm{D} 2 \%$ & $\mathrm{D}_{\text {mean }}$ & V5 & V10 & V20 & V30 \\
\hline LAD & $\mathrm{D} 2 \%$ & $\mathrm{D}_{\text {mean }}$ & V5 & V10 & V20 & V30 \\
\hline Lung & $\mathrm{D} 2 \%$ & $\mathrm{D}_{\text {mean }}$ & V5 & V10 & V20 & V30 \\
\hline Spinal Cord & \multicolumn{6}{|c|}{$\mathrm{D}_{\max } \quad \mathrm{D}_{\text {mean }}$} \\
\hline PTV & & $\mathrm{D}_{\mathrm{m}}$ & & ean & & \\
\hline
\end{tabular}

Each new patient has four KDE predictions, four KDE plans, and two clinical plans. Here we use superscript to identity plan type and use a subscript to identify the image. For example, the original clinical plans were marked as $\operatorname{Plan}_{F B-C T}^{\text {manual }}$ and Plan $_{D I B H-C T}^{\text {manual }}, \mathrm{KDE}$ plans based on FB-CT were marked as $\operatorname{Plan}_{F B-C T}^{F B \bmod e l}$ and $\operatorname{Plan}_{F B-C T}^{D I B H}$ mod $e l$ for two KBMs, plans based on DIBH-CT were marked as Plan ${ }_{D I B H-C T}^{F B \bmod e l}$ and $\operatorname{Plan}_{D I B H-C T}^{D I B H}$ mod $e$ for two KBMs. It is noticeable that in all IMRT plans, the PTV requirements were the same.

\section{Dosimetric comparison}

A paired student's T-test was used to assess the significance of any differences in dose metrics where significance corresponded to a p-value 0.05. Mean doses to the heart, left anterior descending coronary artery (LADCA), and left lung were compared. 


\section{Results}

\section{The performance of the KDE models}

The results (Table2) show that there were no differences between clinical plans

and KDE predictions for both models in the training dataset, confirming that the DVH estimation of the KBM was successful.

Table 2: Summary of OAR doses from IMRT validation, comparing the clinical plan and DVH estimates from its KBM for 40 cases (mean \pm SD).

\begin{tabular}{ccccc}
\hline \hline $\begin{array}{c}\text { Structure } \\
\text { (Average } \\
\text { dose) }\end{array}$ & CT & $\begin{array}{c}\text { Clinical plan } \\
(\mathrm{Gy})\end{array}$ & $\begin{array}{c}\text { KDE } \\
\text { prediction } \\
(\mathrm{Gy})\end{array}$ & $\begin{array}{c}\text { Clinical plan vs. } \\
\text { KDE prediction } \\
\text { (p-value) }\end{array}$ \\
\hline Heart & FB & $1.96 \pm 0.26$ & $1.95 \pm 0.34$ & 0.77 \\
& DIBH & $1.30 \pm 0.31$ & $1.31 \pm 0.23$ & 0.87 \\
LADCA & FB & $16.57 \pm 0.33$ & $16.02 \pm 0.28$ & 0.48 \\
& DIBH & $8.59 \pm 3.73$ & $8.47 \pm 3.08$ & 0.76 \\
Left lung & FB & $5.53 \pm 1.42$ & $5.48 \pm 1.15$ & 0.48 \\
& DIBH & $5.43 \pm 0.59$ & $5.48 \pm 0.60$ & 0.78 \\
\hline \hline
\end{tabular}

Note: Clinical plan represents the plan used in the model training dataset.

\section{The models work in same breath settings}

The results of model performance in the same breath settings for another 10 left-breast patients were presented in Table 3. There was no difference between clinical plan and estimated plan for all structures' mean dose $(\mathrm{p}>0.05)$. Meanwhile, all estimated DVHs can be transferred into deliverable KDE plans. No difference between prediction and KDE plan was observed $(\mathrm{p}>0.05)$. 
Table 3: The comparison of the OAR average doses among the clinical plans, generated plans and the predicted DVHs performed on the same breath settings (mean $215 \pm \mathrm{SD})(\mathrm{Gy})$

\begin{tabular}{ccccccc}
\hline \hline Structure & CT & $\begin{array}{c}\text { Clinical } \\
\text { plan }(G y)\end{array}$ & $\begin{array}{c}\text { KDE } \\
\text { prediction } \\
(\mathrm{Gy})\end{array}$ & $\begin{array}{c}\text { KDE plan } \\
(\mathrm{Gy})\end{array}$ & $\begin{array}{c}\text { Clinical } \\
\text { plan vs. } \\
\text { prediction } \\
(\mathrm{p} \text {-value })\end{array}$ & $\begin{array}{c}\text { prediction } \\
\text { vs. KDE } \\
\text { plan } \\
(\mathrm{p} \text {-value })\end{array}$ \\
\hline Heart & FB & $2.03 \pm 0.38$ & $2.00 \pm 0.37$ & $2.04 \pm 0.38$ & 0.94 & 0.41 \\
& DIBH & $1.17 \pm 0.31$ & $1.27 \pm 0.21$ & $1.29 \pm 0.21$ & 0.14 & 0.29 \\
LADCA & FB & $17.46 \pm 5.01$ & $16.01 \pm 1.08$ & $16.00 \pm 1.20$ & 0.35 & 0.16 \\
& DIBH & $6.70 \pm 4.78$ & $7.66 \pm 1.60$ & $7.79 \pm 1.70$ & 0.37 & 0.06 \\
Left lung & FB & $4.73 \pm 1.62$ & $5.41 \pm 0.90$ & $5.44 \pm 0.99$ & 0.11 & 0.35 \\
& DIBH & $4.88 \pm 1.05$ & $5.48 \pm 0.59$ & $5.46 \pm 0.64$ & 0.14 & 0.23 \\
\hline \hline
\end{tabular}

\section{The FB model works with DIBH-CT}

The result of the FB model works with DIBH-CT was presented in Table 4. The mean dose of which the FB model predicted was higher than the clinical plan $(\mathrm{p}<$ 0.05 for all three structures). By transferring to deliverable KDE plan, the mean dose of the left lung was reduced significantly $(\mathrm{p}=0.01)$.

Table 4: The results of the FB model performed on DIBH-CT in 10 new left-breast IMRT plans (mean \pm SD) (Gy).

\begin{tabular}{cccccc}
\hline \hline Structure & $\begin{array}{c}\text { Clinical } \\
\text { plan (Gy) }\end{array}$ & $\begin{array}{c}\text { KDE } \\
\text { prediction } \\
(\mathrm{Gy})\end{array}$ & $\begin{array}{c}\text { KDE plan } \\
(\mathrm{Gy})\end{array}$ & $\begin{array}{c}\text { Clinical plan } \\
\text { vs. KDE } \\
\text { prediction } \\
\text { (p-value) }\end{array}$ & $\begin{array}{c}\text { KDE } \\
\text { prediction vs. } \\
\text { KDE plan } \\
\text { (p-value) }\end{array}$ \\
\hline Heart & $1.17 \pm 0.31$ & $1.42 \pm 0.26$ & $1.40 \pm 0.26$ & 0.01 & 0.07 \\
LADCA & $6.70 \pm 4.78$ & $12.1 \pm 1.61$ & $11.98 \pm 1.51$ & 0.003 & 0.09 \\
Left lung & $4.88 \pm 1.05$ & $5.61 \pm 1.08$ & $5.24 \pm 0.58$ & 0.0002 & 0.01 \\
\hline \hline
\end{tabular}

\section{The DIBH model works with FB-CT}



the clinical manual plans, the KDE prediction resulted in lower mean doses of the heart and LADCA by $0.24 \pm 0.36 \mathrm{~Gy}(\mathrm{p}=0.02)$, and $4.57 \pm 2.46 \mathrm{~Gy}(\mathrm{p}=0.014)$, respectively. The left lung mean dose of the $\mathrm{KDE}$ prediction was $0.33 \pm 0.99 \mathrm{~Gy}$ higher than the clinical plan $(\mathrm{p}=0.01)$. directly transferred to a deliverable plan.

Table 5: The results of the DIBH model performed on FB-CT in 10 new left-breast IMRT plans (mean \pm SD) $(\mathrm{Gy})$

\begin{tabular}{cccccc}
\hline \hline Structure & $\begin{array}{c}\text { Clinical } \\
\text { plan (Gy) }\end{array}$ & $\begin{array}{c}\text { KDE } \\
\text { prediction } \\
(\mathrm{Gy})\end{array}$ & $\begin{array}{c}\text { KDE plan } \\
(\mathrm{Gy})\end{array}$ & $\begin{array}{c}\text { Clinical plan } \\
\text { vs. KDE } \\
\text { prediction } \\
(\mathrm{p} \text {-value })\end{array}$ & $\begin{array}{c}\text { KDE } \\
\text { prediction vs. } \\
\text { KDE plan } \\
\text { (p-value) }\end{array}$ \\
\hline Heart & $2.03 \pm 0.38$ & $1.79 \pm 0.31$ & $2.09 \pm 0.35$ & 0.02 & 0.04 \\
LADCA & $17.46 \pm 5.01$ & $12.89 \pm 1.85$ & $17.86 \pm 4.09$ & 0.014 & 0.002 \\
Left lung & $4.73 \pm 1.62$ & $5.06 \pm 1.01$ & $5.69 \pm 1.50$ & 0.01 & 0.04 \\
\hline \hline
\end{tabular}

\section{Discussion} respiratory motion. As Schönecker et al. ${ }^{[19]}$ mentioned, DIBH could significantly reduce high dose areas and mean doses to the heart. Our study also proves the earlier results, the great significance of abdominal deep inspiration breath hold in protecting

OARs was shown, during radiation for left-sided breast cancer treatment. However, 
guidance, resulting in more resource-intensive than FB treatments.

It is important to determine early on how much a patient will benefit from DIBH. This research sought to reveal the impact of abdominal breath-holding on knowledge-based treatment planning for breast cancer radiotherapy so that we can guide the application of DIBH more precise before treatment.

In this study, two KDE-based dose prediction models with two different respiratory patterns of FB and DIBH for IMRT treatment were established. The contrast of both FB and DIBH IMRT plans in the original 40 patients, created by manual and KBP method, shows that KBP plans provided at least comparable plan quality compared to clinical ones ( $p>0.05)$.

The further comparative study was performed in another 10 patients similar to the training datasets. There was no significant difference between $\operatorname{Plan}_{F B-C T}^{F B \bmod e l}$ and Plan $_{F B-C T}^{\text {manual }}$ or $\operatorname{Plan}_{D I B H-C T}^{D I B H}$ mod and Plan $_{D I B H-C T}^{\text {manual }}$. The acceptable P-values indicate that our model provides good estimates for DVHs in the same breath settings. A counsel of perfection of Prediction ${ }_{F B-C T}^{D I B H}$ mol $e$ makes unachievable objective targets for $\operatorname{Plan}_{F B-C T}^{D I B H}$ mod $e$. The average OAR dose for Prediction ${ }_{D I B H-C T}^{F B \text { mod } e l}$ was higher than $\operatorname{Plan}_{D I B H-C T}^{\text {manual }}(\mathrm{p}<0.05$ for all clinically relevant structures), and the estimated mean does was slightly higher than the delivered one. It could be due to part of FB model predicted constraint conditions were too relaxed to limit the dose of OARs.

Figure 2 shows one representative example of four situations of the DVHs crossvalidated by two KDE models. The dashed line represents the deliverable plan and the solid line represents the KDE prediction. The DVHs of the heart, LADCA, and 
left-lung were shown in the black lines, red lines, and green lines, respectively.
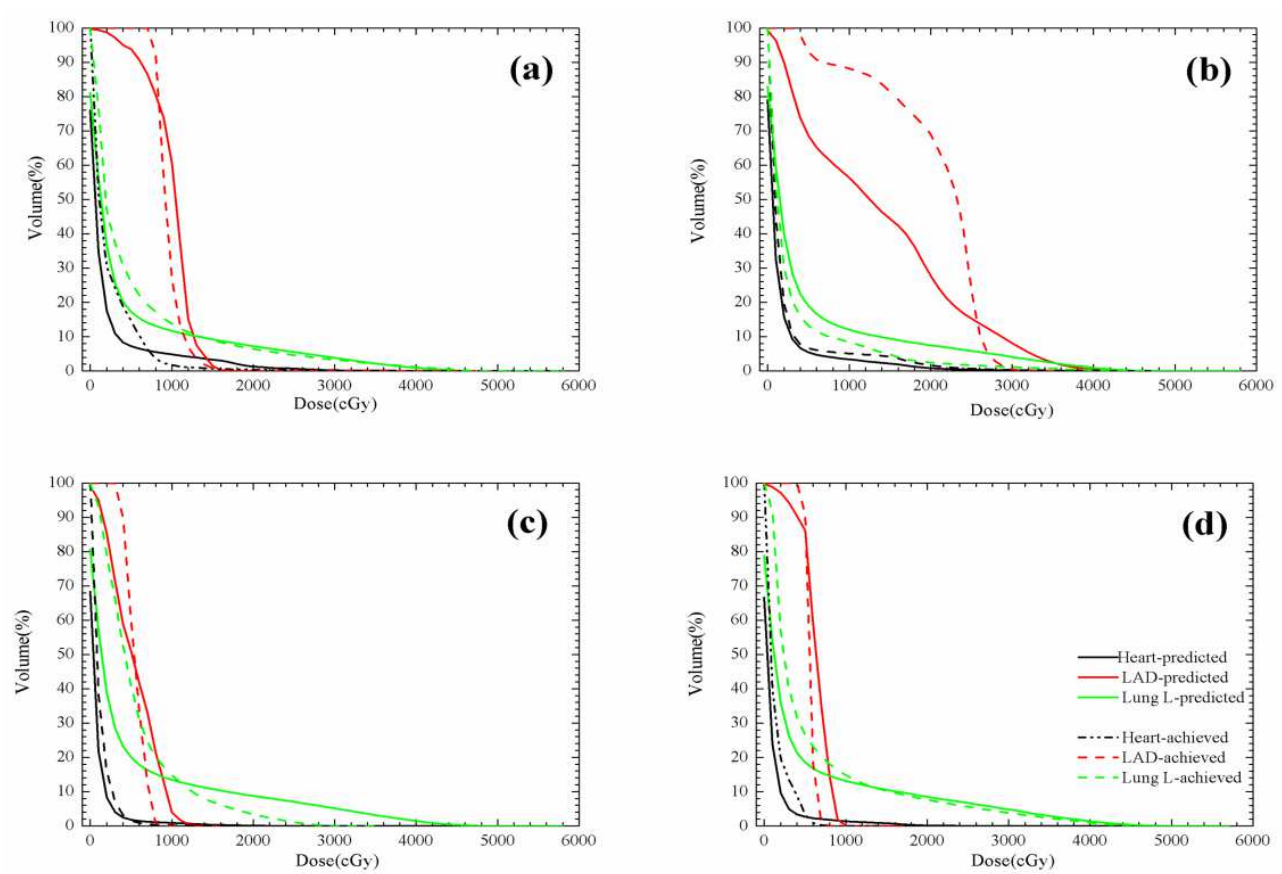

269

270

271

272

273

274

275

276

277

278

279

280

Figure 2: The DVHs of the prediction (solid line) and the deliverable plan (dashed line) for (a)FB model work with FB-CT; (b)DIBH model work with FB-CT; (c) FB model work with DIBH-CT; (d) DIBH model work with DIBH-CT.

All in all, in order to more easily and accurately guide the application of deep inhalation and breath-holding in left breast cancer radiotherapy, we must quantify the protective capacity of DIBH for normal organs. As a method of evaluation, the KBP improves the consistency of all IMRT plans by minimizing the dissimilarity in plan quality due to the variation planner. To create the scoring flow that classifies new patients do the following: put FB-CT and DIBH-CT scans of the left-breast patient into correspondence KDE model, then we can distinguish who would benefit from DIBH more by comparison and analysis.

Evidently selecting the appropriate KBM model in this link is crucial. This 
present work has demonstrated that one KDE model trained with one breath condition may not suitable for all breath conditions. FB model cannot predict DIBH-CT precisely because the establishment of FB model constraints is too loose for DIBH-CT. This may cause plan quality degradation. Meanwhile, there are two problems when using the DIBH model work with FB-CT: one is difficult for plan generation due to the harsh prediction constraints; the other is overtime for plan optimization. However, it is necessary to examine the effect of enlarging the sample size on the model presented in future research.

\section{Conclusions}

The current work developed a KBP model for functional-guided radiotherapy. Modest, but statistically significant, improvements were observed in LAD, functional lung and heart doses of individual model corresponding to CT breathing patterns. Thus, in order to obtain superior performance in a knowledge-based treatment planning, different breathing conditions should be taken into account.

We consider this study innovative because it explains and validates the correlation between classification of the KDE based dose prediction model and breathing maneuvers during left-sided whole-breast irradiation after breast-conserving surgery. It shows that classifying $\mathrm{KDE}$ dose prediction models according to respiratory patterns are indispensable, thus an optimal decision base for automatically making the radiation therapy plan of the marshalling station with computer is supplied. This research, while just a beginning, at least establishes some basic scientific facts 
that could prove useful in future studies on the automatic plan and related conditions.

\section{Declarations}

\section{Ethics approval and consent to participate}

This prospective study was conducted in compliance with the ethical principles of the Declaration of Helsinki and was approved by the Research Ethics Committee of the First Affiliated Hospital, College of Medicine, Zhejiang University. Informed consent was obtained from all individual participants included in the study.

\section{Funding}

This study was supported by grants from Chinese Medicine Research Program of Zhejiang Province (2018KY063), Zhejiang Provincial Key Discipline of Traditional Chinese Medicine (2017-XK-A32), Health and Family Planning Commission of Zhejiang Province in China (2020364043), and Zhejiang Provincial Natural Science (SY19H160009).

\section{Availability of data and materials}

The datasets used and/or analysed during the current study are available from the corresponding author on reasonable request.

\section{Consent for publication}

\section{Not applicable}

\section{Competing interests}

The authors declare that they have no competing interests

\section{Authors' contributions}


JQX: literature search, figures, study design, data analysis, tables, figures, interpretation, manuscript writing and paper revision; JZW, WGH: study design, data collection, manuscript writing and paper revision; FZ, GRY, LYB: study design, radiotherapy planning, data collection, data analysis, interpretation and paper revision; ZJL,SXY: study design, data analysis, interpretation, figures and paper revision; All authors have read and approved the final version of the manuscript.

\section{Acknowledgements}

Not applicable

\section{References}

[1] Steward LT, Gao F, Taylor MA, Margenthaler JA. Impact of radiation therapy on survival in patients with triple-negative breast cancer. Oncol Lett. 2013; 7(2):548-552. [2] Darby SC, McGale P, Taylor CW, Peto R. Long-term mortality from heart disease and lung cancer after radiotherapy for early breast cancer: prospective cohort study of about 300,000 women in US SEER cancer registries. Lancet Oncol.2005;6(8):557-65. [3] Darby SC, Ewertz M, McGale P, et al. Risk of ischemic heart disease in women after radiotherapy for breast cancer. N Engl J Med. 2013; 368(11): 987-98.

[4] Sardaro A, Petruzzelli MF, D'Errico MP, et al. Radiation-induced cardiac damage in early left breast cancer patients: risk factors, biological mechanisms, radiobiology, and dosimetric constraints. Radiother Oncol. 2012; 103(2):133-42.

[5] Pedersen AN, Korreman S, Nyström H, Specht L. Breathing adapted radiotherapy 
of breast cancer: reduction of cardiac and pulmonary doses using voluntary inspiration breath-hold. Radiother Oncol. 2004; 72(1):53-60.

[6] Nissen HD, Appelt AL. Improved heart, lung and target dose with deep inspiration breath hold in a large clinical series of breast cancer patients. Radiother Oncol. 2013; 106(1):28-32.

[7] Aznar MC, Maraldo MV, Schut DA, et al. Minimizing late effects for patients with mediastinal Hodgkin lymphoma: deep inspiration breath-hold, IMRT, or both? Int J Radiat Oncol Biol Phys. 2015; 92(1):169-74.

[8] Beetz I, Schilstra C, van der Schaaf A, van den Heuvel ER, et al. NTCP models for patient-rated xerostomia and sticky saliva after treatment with intensity modulated radiotherapy for head and neck cancer: the role of dosimetric and clinical factors. Radiother Oncol. 2012;105:101-6.

[9] Christianen MEMC, Schilstra C, Beetz I, Muijs CT, et al. Predictive modelling for swallowing dysfunction after primary (chemo) radiation: results of a prospective observational study. Radiother Oncol. 2012; 105:107-14.

[10] Nelms BE, Robinson G, Markham J, Velasco K, Boyd S, Narayan S, et al. Variation in external beam treatment plan quality: an inter-institutional study of planners and planning systems. Pract Radiat Oncol. 2012; 2:296-305.

[11] Berry SL, Ma R, Boczkowski A, Jackson A, Zhang P, Hunt M. Evaluating inter-campus plan consistency using a knowledge based planning model. Radiother Oncol. 2016; 16:10-16.

[12] Fogliata A, Belosi F, Clivio A, Navarria P, Nicolini G, Scorsetti M. On the 
371

372

pre-clinical validation of a commercial model-based optimisation engine: application to volumetric modulated arc therapy for patients with lung or prostate cancer. Radiother Oncol. 2014; 113:385-391.

[13] Petit SF, Wu B, Kazhdan M, Dekker A, et al. Increased organ sparing using shape-based treatment plan optimization for intensity modulated radiation therapy of pancreatic adenocarcinoma. Radiother Oncol. 2012; 102:38-44.

[14] Tol JP, Dahele M, Gregoire V, et al. Analysis of EORTC-1219-DAHANCA-29 trial plans demonstrates the potential of knowledge-based planning to provide patient-specific treatment plan quality assurance. Radiother Oncol. 2019; 130:75-81.

[15] Krayenbuehl J, Norton I, Studer G, Guckenberger M. Evaluation of an automated knowledge based treatment planning system for head and neck. Radiother Oncol. $2015 ; 10: 226$.

[16] Fan J, Wang J, Zhang Z, Hu W. Iterative dataset optimization in automated planning: Implementation for breast and rectal cancer radiotherapy. Med Phys. 2017; 44(6):2515-2531.

[17] Skarpman MJ, Sjolund J. Dose-volume histogram prediction using density estimation. Phys Med Biol. 2015; 60:6923-6936.

[18] Nielsen MH, Berg M, Pedersen AN, et al. Delineation of target volumes and organs at risk in adjuvant radiotherapy of early breast cancer: national guidelines and contouring atlas by the Danish breast Cancer cooperative group. Acta Oncol. 2013;52(4):703-10.

[19] Schönecker S, Walter F, Freislederer P, et al. Treatment planning and evaluation 
393

394

395

396

397

398

399

400

401

402

403

404

405

406

407 408 7

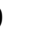

of gated radiotherapy in left-sided breast cancer patients using the catalyst $^{\mathrm{TM}} /$ sentinel $^{\mathrm{TM}}$ system for deep inspiration breath-hold (DIBH). Radiat Oncol. 2016; 11(1):143.

.


Figures

\begin{tabular}{|c|c|c|c|}
\hline $\begin{array}{c}\text { THE WORKFLOW OF THIS } \\
\text { STUDY }\end{array}$ & \multicolumn{3}{|c|}{ Performance eVAluation } \\
\hline \multirow{3}{*}{$\begin{array}{c}\text { Two CT scans } \\
\text { (FB and DIBH) } \\
\text { Model training } \\
\text { (FB and DIBH) }\end{array}$} & & FB model & DIBH model \\
\hline & \multirow{2}{*}{ FB-CT } & $\begin{array}{l}\text { Clinical plan VS. } \\
\text { KDE prediction }\end{array}$ & $\begin{array}{l}\text { Clinical plan VS. } \\
\text { KDE prediction }\end{array}$ \\
\hline & & $\begin{array}{l}\text { KDE plan VS. } \\
\text { KDE prediction }\end{array}$ & $\begin{array}{l}\text { KDE plan VS. } \\
\text { KDE prediction }\end{array}$ \\
\hline prediction & \multirow{2}{*}{ DIBH-CT } & $\begin{array}{l}\text { Clinical plan VS. } \\
\text { KDE prediction }\end{array}$ & $\begin{array}{l}\text { Clinical plan VS. } \\
\text { KDE prediction }\end{array}$ \\
\hline $\begin{array}{l}\text { Performance } \\
\text { evaluation }\end{array}$ & & $\begin{array}{l}\text { KDE plan VS. } \\
\text { KDE prediction }\end{array}$ & $\begin{array}{l}\text { KDE plan VS. } \\
\text { KDE prediction }\end{array}$ \\
\hline
\end{tabular}

\section{Figure 1}

The strategy of knowledge-based planning generated optimization objectives
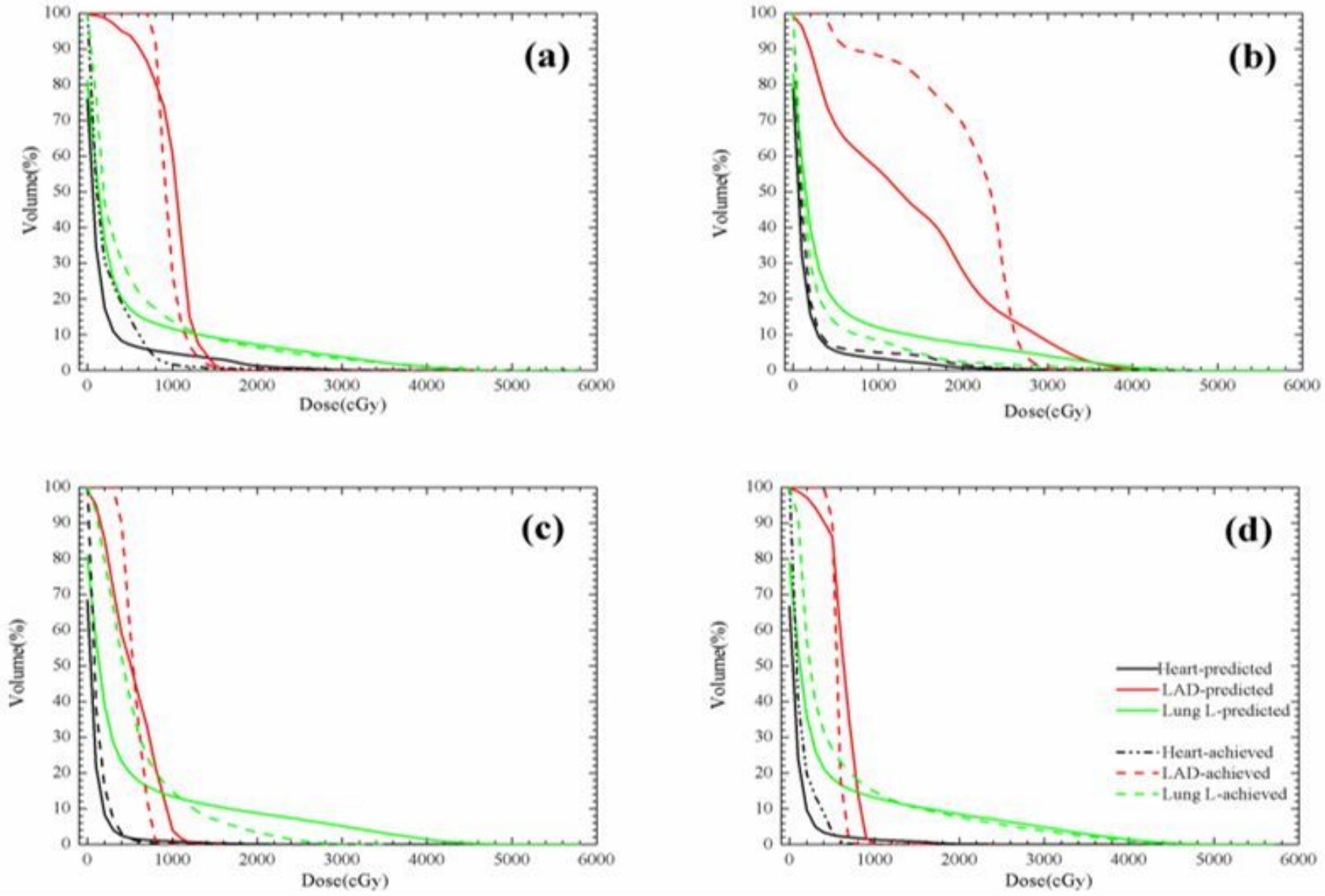
Figure 2

The DVHs of the prediction (solid line) and the deliverable plan (dashed line) for (a)FB model work with FB-CT; (b)DIBH model work with FB-CT; (c) FB model work with DIBH-CT; (d) DIBH model work with DIBHCT. 